\title{
Phase Noise Effects on High Spectral Efficiency Coherent Optical OFDM Transmission
}

\author{
Xingwen Yi, William Shieh, Member, IEEE, and Yiran Ma
}

\begin{abstract}
There are three major advantages for coherent optical orthogonal frequency-division multiplexing (CO-OFDM) transmission using digital signal processing. First, coherent detection is realized by digital phase estimation without the need for optical phase-locked loop. Second, OFDM modulation and demodulation are realized by the well-established computation-efficient fast Fourier transform (FFT) and inverse FFT. Third, adaptive data rates can be supported as different quadrature amplitude modulation (QAM) constellations are software-defined, without any hardware change in transmitter and receiver. However, it is well-known that coherent detection, OFDM, and QAM are all susceptible to phase noise. In this paper, theoretical, numerical, and experimental investigations are carried out for phase noise effects on high spectral efficiency CO-OFDM transmission. A transmission model in the presence of phase noise is presented. By using simulation, the bit error rate floors from finite laser linewidth are presented for CO-OFDM systems with high-order QAM constellations. In the experiments, the phase noise effects from both laser linewidth and nonlinear fiber transmission are investigated. The fiber nonlinearity mitigation based on receiver digital signal processing is also discussed.
\end{abstract}

Index Terms-Coherent communication, common phase error (CPE), dispersion, intercarrier interference (ICI), intersymbol interference (ISI), optical fiber communication, orthogonal frequency-division multiplexing (OFDM), phase estimation, phase noise, quadrature amplitude modulation (QAM), signal processing.

\section{INTRODUCTION}

D IGITAL signal processing has been revolutionizing communication networks. However, the applications of digital signal processing to optical transmissions were hampered by the complexity of high-speed operations. Recent advances of microelectronics, such as analog-to-digital converters (ADCs), digital-to-analog converters (DACs), and digital signal processors, have enabled various applications of digital signal processing of optical signals at $10 \mathrm{~Gb} / \mathrm{s}$ [1], [2]. These applications take advantage of the sophistication and flexibility of software to enhance optical transmissions.

Manuscript received September 12, 2007; revised January 5, 2008. The work of the first author was done when he was with the Victoria Research Laboratory, National ICT Australia, University of Melbourne.

X. Yi was with the Victoria Research Laboratory, National ICT Australia, University of Melbourne, VIC 3010, Australia. He is now with the Department of Electrical and Computer Engineering, University of California, Davis, CA 95616 USA (e-mail: xwyi@ucdavis.edu).

W. Shieh is with the ARC Special Research Centre for Ultra-Broadband Information Networks, Department of Electrical and Electronic Engineering, University of Melbourne, VIC 3010, Australia (e-mail: w.shieh@ee.unimelb.edu. au).

Y. Ma is with the Victoria Research Laboratory, National ICT Australia, University of Melbourne, VIC 3010, Australia.

Digital Object Identifier 10.1109/JLT.2008.919368

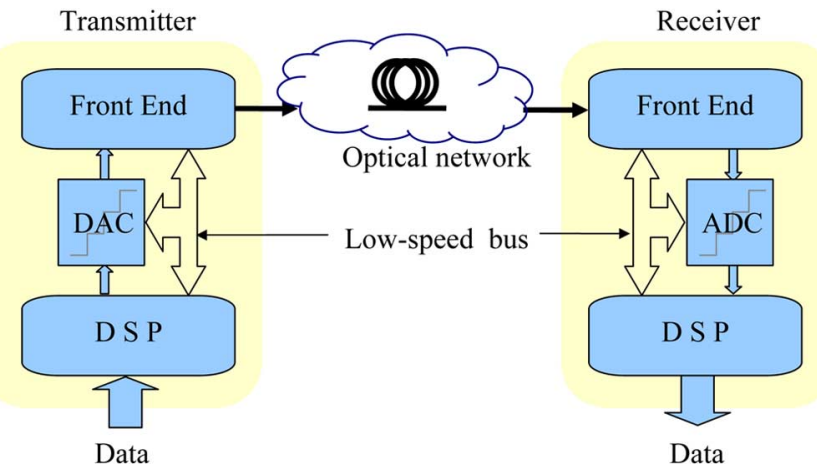

Fig. 1. Conceptual diagram of software-enhanced optical transmission. DSP: digital signal processor.

Fig. 1 shows the conceptual diagram of software-enhanced optical transmission (SEOT). A salient difference to conventional optical transmissions is the presence of DAC/ADC and digital signal processors. For optimization and application purposes, there are low-speed interactions among digital signal processors, DAC or ADC, and the front end in Fig. 1. SEOT is best understood from the development of electronic dispersion compensation (EDC). For instance, for conventional front ends of intensity modulation/direct detection (IM/DD) systems, maximum-likelihood sequence estimation (MLSE) can be used [2]; for an optical in-phase/quadrature (IQ) modulator and direction detection, precompensation can be used [1]; for a coherent detection front end, digital phase estimation can be used to replace conventional optical phase-locked loops (OPLL) [3]; for an optical IQ modulator and coherent detection front end, coherent optical OFDM (CO-OFDM) can be realized [4]. In these examples, their front ends are quite different, but they all take advantage of digital signal processing to achieve significant performance enhancements of chromatic dispersion tolerance. However, the capabilities of SEOT are far beyond dispersion compensation.

CO-OFDM is a typical example of SEOT to leverage the capabilities of digital signal processing [5]. In a generic CO-OFDM system, the transmitter front end includes an optical IQ modulator and the receiver front end includes a coherent receiver. Consequently, the transmitter fully controls the optical carrier and the receiver accesses all the optical information, which significantly enhance the capabilities of digital signal processing. Its detailed architecture, digital signal processing, and system performance have been reported in [4], [6]-[8]. By running different software in a digital signal processor, CO-OFDM presents numerous flexibilities and advantages.

- Without any hardware change, each subcarrier can have different modulation formats, including quadrature ampli- 
tude modulation (QAM). In essence, the channel data rate can be adjusted according to the optical channel condition.

- Some hardware imperfections of the front end can be addressed by software, such as the laser phase drift [7] and the nonlinear transfer function of the optical modulator [9].

- The transmission channel characteristics can be monitored by means of receiver signal processing [10]. Subsequently, all the OFDM parameters, from subcarrier number to individual subcarrier modulation format, can be optimized [11].

- The fiber linear dispersion and nonlinear phase noise can be mitigated by digital signal processing [8].

Note there are some other varieties of optical OFDM [12], [13], which may have simpler front ends. However, they do not have all the advantages of CO-OFDM owing to coherent detection.

Unfortunately, it is commonly accepted that coherent detection, OFDM, and QAM are all susceptible to phase noise. Prior to this work, the discussions of phase noise on coherent detection and QAM are focused on single-carrier optical transmission systems and most of them are for OPLL [14], [15]. Phase noise on OFDM is mainly in the context of wireless transmissions [16]-[19]. Therefore, it is of great interest to investigate phase noise on CO-QAM-OFDM transmissions.

This paper is organized as follows. In Section II, a CO-OFDM transmission model in the presence of phase noise is presented with an emphasis on digital signal processing, and the terminologies in this paper are introduced. In Section III, the bit error rate (BER) performance limited by additive white Gaussian noise (AWGN) and laser phase noise is separately investigated. The BER performance in AWGN is presented in terms of optical signal-to-noise ratio (OSNR). The BER floors of different M-QAM-OFDM contributed by laser phase noise are presented with extensive simulations. In Section IV, phase noise effects on CO-QAM-OFDM systems are investigated by two experiments, in which the effective bit rates of 16-QAM-OFDM and 64-QAM-OFDM are 10.59 and 15.89 $\mathrm{Gb} / \mathrm{s}$, respectively. Without polarization multiplexing, their spectral efficiencies are 2.8 and $4.2 \mathrm{bit} / \mathrm{s} / \mathrm{Hz}$, correspondingly. In the first experiment, phase noise effects from laser phase noise on 16-QAM-OFDM and 64-QAM-OFDM are experimentally measured and compared to the simulation results. In the second experiment, nonlinear phase noise effects from a single-span transmission with high launch power and a multi-span long haul transmission, up to $1000 \mathrm{~km}$, are shown and discussed. The fiber nonlinearity mitigation based on receiver digital signal processing is also discussed.

\section{TRANSMISSION MODEL OF CO-OFDM USING DigiTAL SigNAL PROCESSING}

There are many varieties of coherent detection, OFDM, and QAM. Consequently, phase noise effects can vary significantly for them. In this work, coherent detectionis realized through digital phase estimation and compensation by pilot-aided phase estimation described in [7]. Data-aided phase estimation is not considered here due to the implementation difficulties when QAM formats are used [20]. M-QAM stands for square QAM with gray coding and rectangle decision areas. M-QAM may not

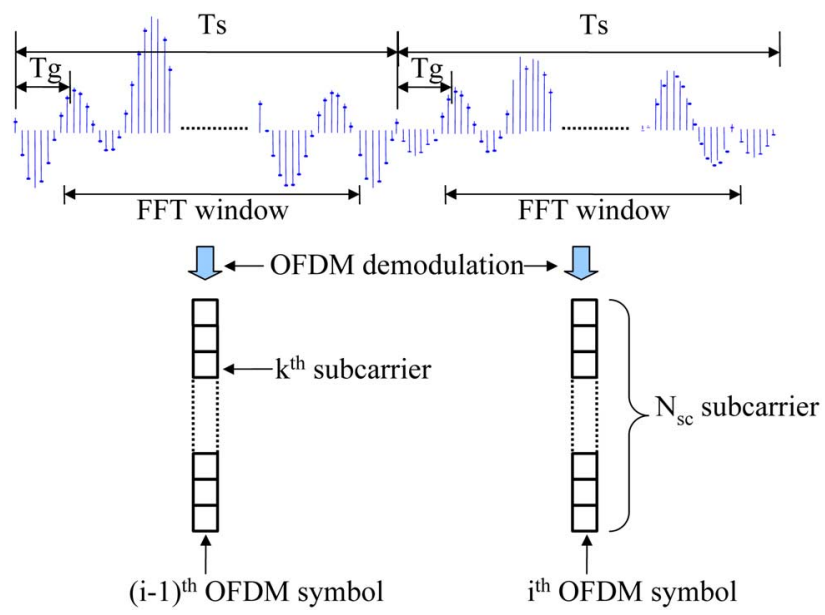

Fig. 2. OFDM demodulation.

be the optimum constellation in the presence of phase noise, but it is the most practical one, especially for higher order constellations [20], [21]. The principle of OFDM transmission is welldiscussed in [11] and [22], and the architecture of CO-OFDM is detailed in [4] and [5]. The following description aims to introduce the transmission model using digital signal processing and the terminologies used in this paper.

The receiver front end in this work is a coherent optical receiver and digital signal processing starts from a sampled sequence with an ADC at a time interval of $T$. When heterodyne coherent detection is used, the sampled signal needs to be software down-converted to baseband, which involves intermediate frequency (IF) synchronization [4]. Fig. 2 shows OFDM demodulation by fast Fourier transform (FFT). The time duration of one OFDM symbol and guard interval are $T_{s}$ and $T_{g}$, respectively. The guard interval is the so-called cyclic prefix (CP) to eliminate intersymbol interference (ISI). The CP ratio, $r_{\mathrm{cp}}=T_{g} / T_{s}$, is used to describe the overhead of CP since it carries no additional information. By digital signal processing, the FFT window is located and $N_{s c}$ sampling points are subsequently selected to perform FFT, or OFDM demodulation. FFT length is $N_{s c}$, which is the total number of subcarrier. The sampling speed is $1 / T$, and therefore the FFT window period is $T_{s}-T_{g}=N_{s c} T$. In practice, part of subcarriers, so-called virtual subcarriers, are set to zeros to obviate the aliasing noise during sampling or to facilitate electrical filtering.

By using digital phase estimation, the coherent detection is realized after digital phase estimation and compensation, even though the local laser is free-running. As a result, the sampling sequence before OFDM demodulation includes laser phase noise. The transmission model of CO-OFDM can be found in [7], which is a parallel multiple subcarrier transmission model. By assuming a perfect FFT window synchronization, the CO-OFDM transmission model is given by

$$
\begin{aligned}
& y_{i k}=x_{i k} h_{k} e^{j \Phi_{i}^{c}}+\varepsilon_{i k}+n_{i k} \\
& e^{j \Phi_{i}^{c} \triangleq} \frac{1}{N_{s c}} \sum_{n} e^{j \Phi(n)}
\end{aligned}
$$

where $x_{i k}$ and $y_{i k}$ are the M-QAM modulated data of the $k$ th subcarrier in the $i$ th OFDM symbol before and after transmis- 
sion, $h_{k}$ is the transmission channel response at $k$ th subcarrier, $\Phi(n)$ is the laser phase noise sampling sequence with the $T$ time interval, $n_{i k}$ is complex white Gaussian noise. $\varepsilon_{i k}$ is intercarrier interference (ICI) and is generally treated as white Gaussian noise when a large number of OFDM subcarriers are used. The expression of $\varepsilon_{i k}$ can be found in [17]. $\Phi_{i}^{c}$ is common phase error (CPE) and is contributed either by the laser phase drift or the phase noise during optical fiber transmissions. Note the transmission channel for each subcarrier starts from OFDM modulation (inverse FFT) in transmitter and terminates at OFDM demodulation (FFT) in receiver. As a result, the transmission channel response $h_{k}$ includes not only the optical channel but also the front ends and ADC/DAC.

The laser phase noise sequence $\Phi(n)$ is generally treated as a zero-mean Gaussian process. For the small phase change, $\Phi_{i}^{c}$ is linearly related to $\Phi(n)$, and consequently is also zero-mean Gaussian process. The variance of phase difference is related to laser linewidth and used to indicate the strength of phase noise, defined as

$$
\sigma_{c}^{2}=\frac{1}{N_{f}} \sum_{i}\left(\Phi_{i}^{c}-\Phi_{i-1}^{c}\right)^{2}=2 \pi \beta T_{s}
$$

where $\beta$ is the combined linewidth of transmitter and receiver lasers, $N_{f}$ is the number of OFDM symbols used to calculate the variance.

In (1), CPE $\Phi_{i}^{c}$ is independent of subcarrier and can be estimated and compensated by using several pilot subcarriers described in [7]. The channel response in optical transmission is slowly varying and therefore $h_{k}$ is independent of OFDM symbol within a block of OFDM symbols. In this work, some OFDM symbols are used as the preamble to estimate $h_{k}$. After CPE and transmission channel response estimation, the recovered transmitted data are

$$
\begin{aligned}
x_{i k}^{r} & =y_{i k} \cdot e^{-j \Phi_{i}^{c}} \cdot h_{k}^{*} /\left|h_{k}\right|^{2} \\
& =x_{i k}+\left(\varepsilon_{i k}+n_{i k}\right) \cdot e^{-j \Phi_{i}^{c}} \cdot h_{k}^{*} /\left|h_{k}\right|^{2} .
\end{aligned}
$$

The recovered data of each subcarrier are corrupted by a noise term. With some assumptions, the performance after compensation may be approximated [18], [19], but the accurate evaluation has to employ numeric simulations, especially for high-order QAM formats.

For convenience, the mathematical symbols used in this paper are summarized in Table I.

\section{BER PERformance Due to Gaussian NOISE AND LASER PHASE NOISE}

In CO-OFDM transmission systems, three main noise sources are additive white Gaussian noise from amplified spontaneous emission (ASE) noise, laser phase noise from transmitter and receiver lasers, and nonlinear phase noise from optical fiber transmissions. The BER performance is separately discussed for the first two noises in this section. The third noise is discussed in Section IV.

\section{A. BER Performance in AWGN}

The BER performance of M-QAM-OFDM has been documented in [11], [20], and [22] in terms of SNR (per bit
TABLE I

LisT OF MATHEMATICAL SyMbOLS

\begin{tabular}{ll}
\hline$T$ & sampling time interval of ADC \\
$T_{s}$ & time duration of OFDM symbol \\
$T_{g}$ & time interval of cyclic prefix \\
$r_{c p}$ & ratio of $T_{g}$ to $T_{s}$ \\
$i$ & index of OFDM symbol \\
$k$ & index of OFDM subcarrier \\
$x_{i k}$ & transmitted data at $i$ th OFDM symbol and $k$ th subcarrier \\
$y_{i k}$ & received data at $i$ th OFDM symbol and $k$ th subcarrier \\
$h_{k}$ & optical channel response at $k$ th subcarrier \\
$N_{s c}$ & number of subcarriers \\
$N_{s c u}$ & number of used subcarriers \\
$N_{p}$ & number of pilot subcarriers \\
$\Phi(n)$ & laser phase drift sequence \\
$\Phi_{i}^{c}$ & common phase error sequence \\
$\beta$ & combined laser linewidth of transmitter and receiver lasers \\
$\Gamma$ & ratio of combined laser linewidth to symbol rate \\
$\sigma_{c}^{2}$ & phase variance of $\Phi_{i}^{c}$ \\
$n_{i k}$ & additive complex white Gaussian noise \\
$\varepsilon_{i k}$ & inter-carrier interference \\
$M$ & constellation points of square QAM \\
$R_{s}$ & SNR per symbol \\
$R_{b}$ & SNR per bit \\
\hline
\end{tabular}

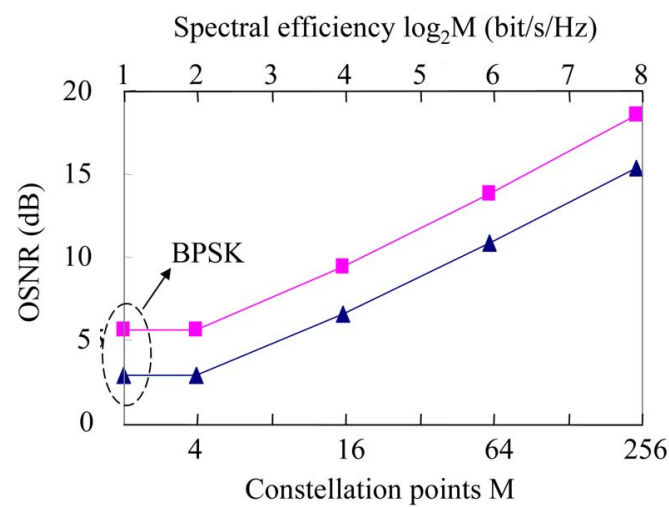

Fig. 3. Required OSNR at $10^{-3}$ and $10^{-5}$ BER for CO-QAM-OFDM at a fixed total bit rate of $10 \mathrm{~Gb} / \mathrm{s}$. The curve with triangles is for $10^{-3} \mathrm{BER}$, and the curve with squares is for $10^{-5}$ BER.

unless otherwise stated). However, OSNR is widely used in the optical community. If the total transmission bit rate is fixed, it is of interest to know the BER performance of different M-QAM-OFDM in terms of OSNR. Note the BER performance versus SNR is bit-rate independent whereas the BER performance versus OSNR depends on bit-rate and spectral efficiency because OSNR is defined in 0.1-nm bandwidth. In this work, SNR and OSNR are linearly related due to coherent detection.

Fig. 3 shows the required OSNR at $10^{-3}$ and $10^{-5}$ BER for CO-QAM-OFDM systems at a fixed $10-\mathrm{Gb} / \mathrm{s}$ total bit rate with ideal conditions. For simplicity, there is no guard interval, or $T_{g}=0$, and the detection corresponds to direct down-conversion or homodyne detection. The BER performance of M-QAM-OFDM is calculated by (12) in the Appendix. The BER performance of BPSK is also included. As shown in Fig. 3, the lowest OSNR is achieved with 4-QAM-OFDM and BPSK-OFDM. Compared with conventional IM/DD systems [2], 16-QAM-OFDM still has a better OSNR performance. Therefore, both 4-QAM-OFDM and 16-QAM-OFDM are favorable for optical transmissions. Note the results in Fig. 3 
are linearly scalable to higher bit-rate systems. For instance, the OSNR curves can be shifted $6 \mathrm{~dB}$ upward for $40-\mathrm{Gb} / \mathrm{s}$ systems.

In practical CO-QAM-OFDM implementations, cyclic prefix and pilot subcarriers are necessary overheads and require more bandwidth. Consequently, there is an OSNR penalty in addition to the results in Fig. 3. The penalty in the linear scale can be written as

$$
P e n=\frac{N_{s c u}}{\left(N_{s c u}-N_{p}\right)\left(1-r_{\mathrm{cp}}\right)}
$$

where $N_{s c u}$ is used subcarriers for data transmission and pilot, $N_{p}$ is the number of pilot subcarriers.

\section{B. Simulation Results of Laser Phase Noise Effects}

The laser phase noise is characterized by its linewidth. Therefore, the system design of CO-OFDM systems has to take account of laser linewidth. Specifically, the constraints in terms of the number of subcarriers $N_{s c}$ and the constellation points $M$ need to be investigated for a specific laser linewidth. On the other hand, it may be advantageous to have large values of these parameters for ISI tolerance and high spectral efficiency. The trade-off of these two considerations has to be made to choose appropriate $N_{s c}$ and $M$ for a specific application, which is the aim of this section.

$T_{g}$ is used to combat ISI but wastes bandwidth. Therefore, $r_{\mathrm{cp}}$ should be small to limit the overhead. In this work, $r_{\mathrm{cp}}$ is fixed as (1)/(17), and therefore $T_{g}$ is proportional to $N_{s c} \cdot N_{s c}$ is related to tolerable chromatic dispersion $D_{t}$ and ratio of combined laser linewidth to subcarrier symbol rate $\Gamma$ as [5]

$$
\begin{gathered}
\frac{c}{f^{2}} \frac{\left|D_{t}\right|}{T} \leq T_{g}=\frac{r_{\mathrm{cp}} N_{s c} T}{1-r_{\mathrm{cp}}} \\
\Gamma=\beta T_{s}=\frac{\beta N_{s c} T}{1-r_{\mathrm{cp}}}
\end{gathered}
$$

where $c$ is light speed and $f$ is optical carrier frequency. Note $(1) /(T)$ is the total bandwidth of $N_{s c}$ subcarriers and $(1) /\left(T_{s}\right)$ is the subcarrier symbol rate. The ISI source is limited to chromatic dispersion in this work. The PMD induced ISI is simply a substitution of the left side by maximum DGD in (6). The ratio of combined laser linewidth to subcarrier symbol rate defined by (7) determines the BER performance of M-QAM-OFDM.

As per the discussion of (4), the evaluation of BER performance of M-QAM-OFDM in the presence of phase noise is complicated by two facts: the noise distribution on each constellation point is non-Gaussian; the same phase noise has different effect for different constellation points. Therefore, extensive Monte Carlo simulations are carried out. To isolate the phase noise effect, no ASE noise is added in the simulations.

Fig. 4 shows the BER floors of varying laser linewidth and QAM constellation. The OFDM frame and digital signal processing of the simulation are almost identical to those used in the following experiment. In simulation, the OFDM trace from the transmitter program is directly sent to the receiver program. In our experiment, the OFDM trace from the transmitter program is sent to the waveform generator, and the sampling trace from the scope is fed to the receiver program. The laser phase noise is simulated by white Gaussian frequency noise. Each BER point

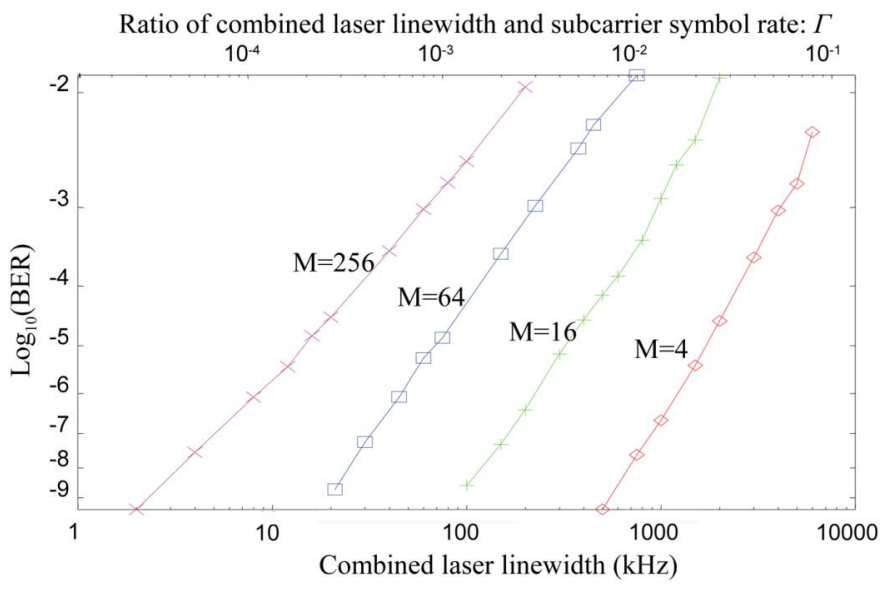

Fig. 4. BER floors of M-QAM-OFDM from finite laser linewidth. Subcarrier number is 128 . Symbol rate for each subcarrier is $73.5 \mathrm{MSymbol} / \mathrm{s}$.

TABLE II

REQUIRED LASER LINEWIDTH FOR M-QAM-OFDM

\begin{tabular}{|c|l|l|}
\hline $\mathrm{M}$ & $\begin{array}{l}\beta \quad(\mathrm{kHz}) \quad / \quad \Gamma \\
\left(\mathrm{BER}=10^{-3}\right)\end{array}$ & $\begin{array}{l}\beta \quad(\mathrm{kHz}) \\
\left(\mathrm{BER}=10^{-5}\right)\end{array}$ \\
\hline 4 & $4100 / 5.6 \times 10^{-2}$ & $1700 / 2.3 \times 10^{-2}$ \\
16 & $956 / 1.3 \times 10^{-2}$ & $322 / 4.4 \times 10^{-3}$ \\
64 & $222 / 3.0 \times 10^{-3}$ & $69.6 / 9.5 \times 10^{-4}$ \\
256 & $61.1 / 8.3 \times 10^{-4}$ & $14.6 / 1.9 \times 10^{-4}$ \\
\hline
\end{tabular}

is recorded where there are more than 100 errors. The main parameters are $N_{s c}=128, r_{\mathrm{cp}}=(1) /(17)$. The symbol rate for each subcarrier is fixed to $73.5 \mathrm{MSymbol} / \mathrm{s}$. The rest of the parameters will be described in the experimental setup description in Section IV.

Given a BER floor, $\Gamma$ or laser linewidth of different CO-QAM-OFDM can be found in Fig. 4. At the same time, the number of subcarriers may be changed according to (6) and (7). Table II reports the values with $73.5 \mathrm{MSymbol} / \mathrm{s}$ for each subcarrier.

From Table II, 16-QAM-OFDM requires a combined laser linewidth of $322 \mathrm{kHz}$ at $10^{-5} \mathrm{BER}$. Since the commercially available semiconductor lasers can have linewidth about $100 \mathrm{kHz}$ [23], coherent optical 16-QAM-OFDM seems feasible.

OSNR penalty has been widely used to evaluate the system performance. Therefore, it is of interest to study OSNR penalty due to laser phase noise. Although accurate evaluation relies on numeric simulation, there are a few analytical estimation methods [16], [20]. An approximation in linear scale is given below [16]

$$
\begin{aligned}
\text { Pen } & =1+\sigma_{c}^{2} R_{s} \\
& =1+2 \pi \beta T_{s} R_{s} \\
R_{s} & =k \cdot \mathrm{OSNR}
\end{aligned}
$$

where SNR per symbol $R_{s}$ is linearly dependent on OSNR by the coefficient $k$ because of coherent detection. Under ideal conditions, $k$ is the ratio of $12.5 \mathrm{GHz}(0.1-\mathrm{nm}$ OSNR resolution) to the receiver electrical bandwidth. In practical systems, $k$ depends on various detection parameters but can be obtained empirically since the parameters are generally unchanged. SNR per 


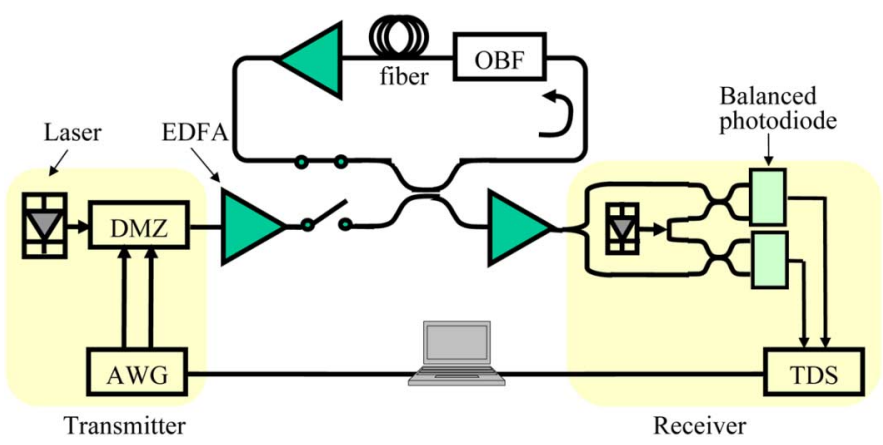

Fig. 5. Experimental setup. Dual Mach-Zehnder modulator. Arbitrary waveform generator. Time-domain scope. Optical band-pass filter.

symbol $R_{s}$ is related to SNR per bit $R_{b}$ by (11) in the Appendix. Note the OSNR penalty depends on the OSNR value and is proportional to the OSNR value, which means that phase noise is more problematic for the high-order modulation that requires high OSNR.

\section{EXPERIMENTAL INVESTIGATIONS OF PHASE NoISE EFFECTS}

The phase noise in CO-QAM-OFDM transmissions are mainly contributed from laser sources, optical amplifiers, and nonlinear effects in optical fibers. The ASE-induced phase noise is well-behaved as AWGN and is not discussed here. Therefore, the experimental investigations are separated into two parts for laser phase noise and nonlinear phase noise, respectively. This section is the extension of the previous work in [24]. The main addition is the discussion on the effectiveness of fiber nonlinear phase noise mitigation based on receive signal processing.

\section{A. Experimental Setup}

The experimental setup is similar to the previous work [8], and Fig. 5 is a simplified illustration with an emphasis on hardware structure. Due to the advantage of SEOT, the experimental structures of transmitter and receiver are for any M-QAM-OFDM. Recall the conceptual diagram in Fig. 1. The transmitter front end in this experiment includes an external cavity laser, an optical IQ modulator, and its radio-frequency (RF) amplifiers. The receiver front end includes another external cavity laser, two polarization beam splitters (not shown in Fig. 5) and two optical couplers, and two balanced receivers with built-in RF amplifiers. The DAC is emulated by an arbitrary waveform generator (AWG) operated at $10 \mathrm{GSa} / \mathrm{s}$ per channel while the ADC is emulated by a time-domain sampling scope (TDS) at $20 \mathrm{GSa} / \mathrm{s}$ due to the heterodyne detection. The DAC in AWG and ADC in TDS both have eight-bit resolution. The digital signal processors are substituted with a computer.

All the major changes to the previous work are in software to accommodate the M-QAM-OFDM format. M-QAM modulation uses gray coding and demodulation uses rectangular decision areas. The pilot-aided phase estimation is based on the description in [7] with a modification of QAM modulated pilot subcarriers.

In this work, 128 OFDM subcarriers are used, $N_{s c}=128$ (or FFT length), but only 36 subcarriers are used for data transmission. Eight more subcarriers, $N_{p}=8$, are reserved as pilot subcarriers for laser phase drift estimation [7], and the

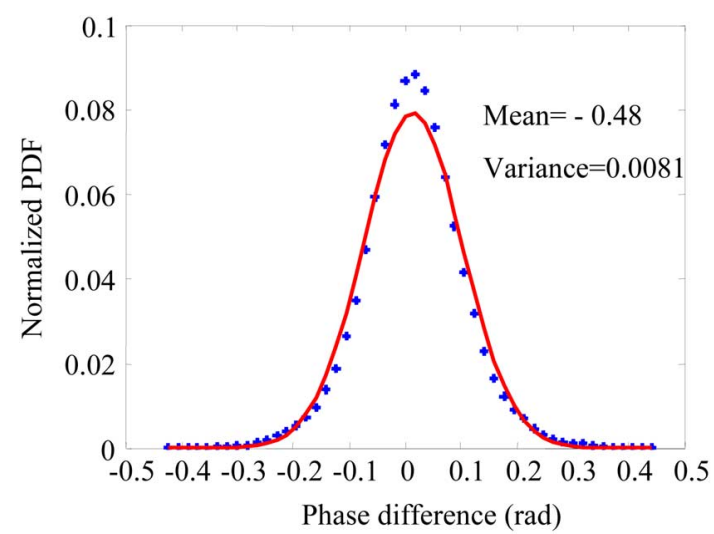

Fig. 6. Normalized histogram of phase difference of common phase error in the time interval of $14.4 \mathrm{~ns}$. The continuous curve is from Gaussian fitting.
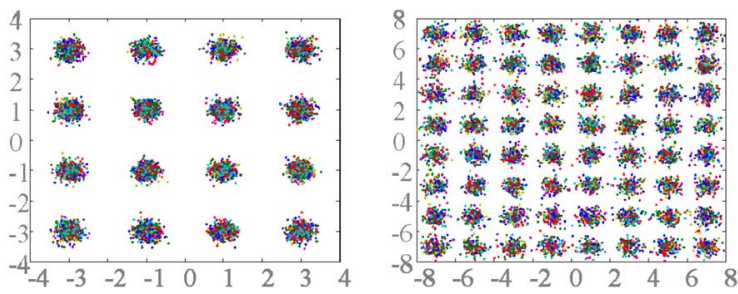

(a)

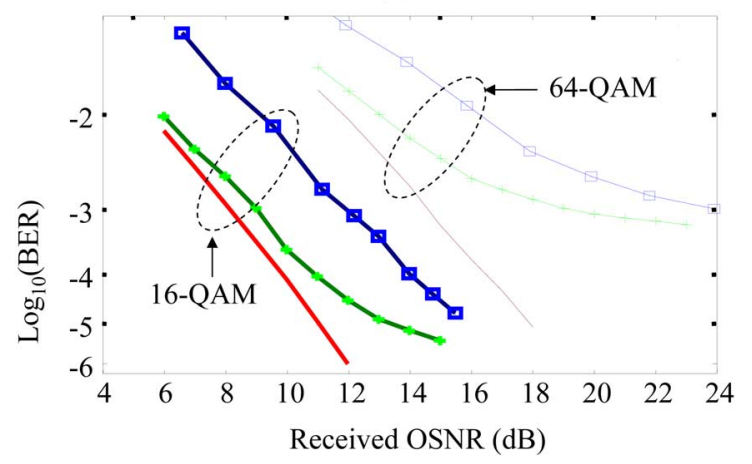

(b)

Fig. 7. (a) Constellations of 16-QAM-OFDM and 64-QAM-OFDM. (b) Back-to-back transmission performance. The solid lines without symbols are for zero-linewidth lasers. The curves with crosses are simulated results with $101-\mathrm{kHz}$ combined linewidth. The curves with squares are experimental results.

remaining subcarriers are padded with zeros to avoid aliasing noise. The time duration of one OFDM symbol is $T_{s}=13.6 \mathrm{~ns}$, and the guard interval is $T_{g}=0.8 \mathrm{~ns}$. Note the 44 subcarriers only occupy $3.5-\mathrm{GHz}$ bandwidth, and the 36 data subcarriers correspond to $2.65 \mathrm{GSymbol} / \mathrm{s}$. Therefore, the effective bit rates of 16-QAM-OFDM and 64-QAM-OFDM are 10.59 and $15.89 \mathrm{~Gb} / \mathrm{s}$, respectively. Without polarization multiplexing, their spectral efficiencies are 2.8 and $4.2 \mathrm{bit} / \mathrm{s} / \mathrm{Hz}$, correspondingly. Consequently, CO-QAM-OFDM can achieve high spectral efficiency. In addition, since 44 subcarriers are less than half of 128 subcarriers, the system can also be considered as oversampling, which is used to avoid the aliasing noise.

\section{B. Laser Linewidth Effects}

The recirculating loop in Fig. 5 is bypassed to investigate the laser linewidth effects. Fig. 7(a) shows the constellations from 
14400 QAM symbols. The SNR per symbol is about $25 \mathrm{~dB}$ for both constellations. The combined linewidth $\beta$ of transmitter and receiver lasers can be estimated by (3). Fig. 6 shows the histogram of estimated laser phase drift. The measured results are close to a Gaussian fit. From several measurements, the combined laser linewidth $\beta$ is estimated as about $101 \mathrm{kHz}$, which is almost half of the specification of the lasers. The estimated linewidth is subsequently used in the simulation, and the resultant BER curve is compared with the experiment results in Fig. 7(b). The BER curves of ideal lasers with zero-linewidth are also included. Compared with the results in Fig. 3, the ideal laser bounds in Fig. 7(b) increase about $4.1 \mathrm{~dB}$ to account for the heterodyne detection and the OSNR penalty described by (5). The heterodyne detection in this work is without image rejection filters, and it is expected that the system performance can be improved by $3 \mathrm{~dB}$ if narrow optical filters are applied to reject the image noise. The received OSNR is $11.1 \mathrm{~dB}$ at $10^{-3}$ BER for 16-QAM-OFDM from the experimental result. Note this result shows that 16-QAM-OFDM can achieve similar OSNR performance to conventional IM/DD systems [2]. 64-QAM-OFDM is strongly limited by the laser phase noise and has an apparent BER floor, confirmed by both the simulation and experiment. In Fig. 7(b), the experimental curves become closer to the simulation curves for the higher OSNR. The main reason is that the estimated $101-\mathrm{kHz}$ linewidth includes small frequency jitters, so-called $1 / f$ frequency noise, which is also confirmed by the imperfect Gaussian curve fitting in Fig. 6. This kind of noise leads to overestimation of the laser linewidth but does not degrade BER in high-bit-rate systems [25]. Therefore, the actual combined linewidth of the two lasers in this work should be smaller than $101 \mathrm{kHz}$. In other words, the simulation BER curves should be lower.

In Table II, it has been predicted that at $10^{-5} \mathrm{BER}, 16-\mathrm{QAM}$ OFDM and 64-QAM-OFDM (73.5 MSymbol/s per subcarrier) require a combined laser linewidth of 322 and $69.6 \mathrm{kHz}$, respectively. The prediction is partially verified in Fig. 7(b), which shows that 16-QAM-OFDM can have a BER lower than $10^{-5}$ whereas 64-QAM-OFDM cannot.

\section{Nonlinear Phase Noise From Optical Fiber Transmissions}

From Fig. 7(b), 64-QAM-OFDM is strongly limited by the laser phase noise. Therefore, only 16-QAM-OFDM is used to investigate the nonlinear phase noise from optical fiber transmissions in this work. The nonlinear optical fiber transmissions include two scenarios: 1) single-span transmission with high launch optical power; and 2) multi-span transmission with long distance. The two scenarios are investigated in the following two experiments.

In the single-span transmission, 50-km standard single-mode fiber (SSMF) is used to investigate the nonlinear phase noise arising from high launch powers. The experimental setup is based on Fig. 5, but the recirculating loop is replaced with the 50-km SSMF fiber. Fig. 8 shows the Q-factor and phase drift variance versus launch optical power. The phase drift variance is defined in (3). Fig. 8 also shows the nonlinear phase noise mitigation results. The mitigation method is the same as described in [8].

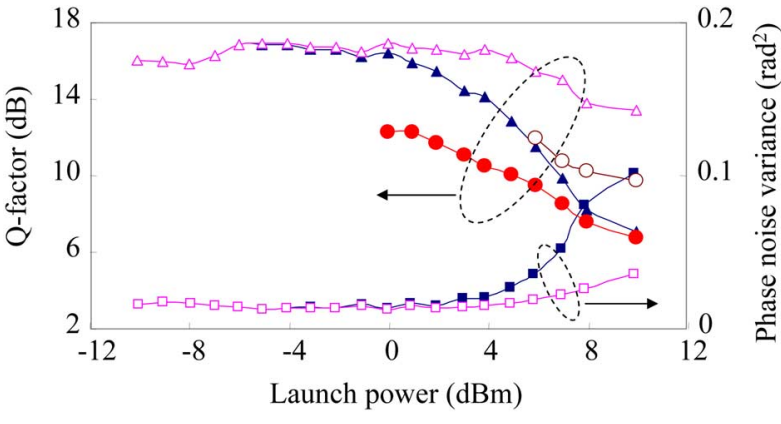

Fig. 8. Q-factor and phase drift variance of 16-QAM-OFDM versus launch power. The curves with open symbols are after fiber nonlinearity mitigation. The curves with triangles and circles are monitored-Q and calculated-Q, respectively.

For the high-order M-QAM, the Q-factor is converted from BER by (14) in the Appendix. There are two ways to calculate the BER, consequently Q-factor. The first way is based on AWGN assumption and calculated from SNR by (12) in the Appendix, which is essentially the constellation spreading [10], [21]. Note this calculation method inherits the well-documented QAM performance to calculate the Q-factor; otherwise it would involve the discussion of probability density functions and decision areas [21], [26]. This method also guarantees a fixed mapping between Q-factor and BER in AWGN, which is independent of modulation format and is the same as conventional optical transmission systems [26]. The second way is the result of data decision and error computation. The resultant two Q-factors are both plotted in Fig. 8. For clarity, the Q-factor from the BER decision is named calculated-Q, and the Q-factor from noise spreading, monitored-Q.

In Fig. 8, the Q-factors barely change from -6 to $0 \mathrm{dBm}$ despite the varying received OSNR. This is because the system performance is capped by the electrical components. When the launch power is higher than $0 \mathrm{dBm}$, the phase drift variance begins to increase while the $\mathrm{Q}$-factor decreases. The difference between two Q-factors is obvious, but they both show the correct trend. It is apparent that the system is corrupted by the phase noise and cannot be approximated by AWGN. The Q-factor based on AWGN overestimates the system performance. The accurate system performance analysis needs to include the phase drift variance. However, the validity of (8) is compromised by the fact that phase noise and Gaussian noise are not distinguishable in this experiment. The performance evaluation of CO-QAM-OFDM in the presence of strong phase noise is under further study. The calculated-Q is not shown for the low launch power because it is difficult to obtain the meaningful BER when it is low. For the same reason, Q-factor is still used in the following experiment, even though it may overestimate the system performance. The fiber nonlinearity mitigation improves the system performance significantly, manifested by the difference between the curves with solid and open symbols. The phase drift variance is reduced and Q-factor is increased about $3 \mathrm{~dB}$. The tolerable launch power into fiber is also shifted to about $5 \mathrm{dBm}$.

In Fig. 8, the optimum launch power is about $3 \mathrm{dBm}$ per channel with negligible fiber nonlinearity. Therefore, the op- 


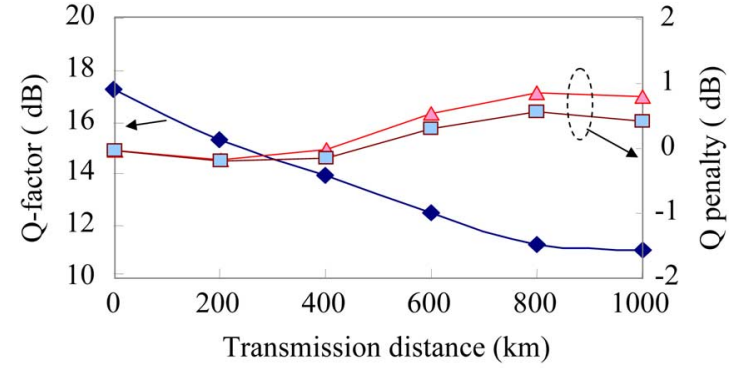

(a)

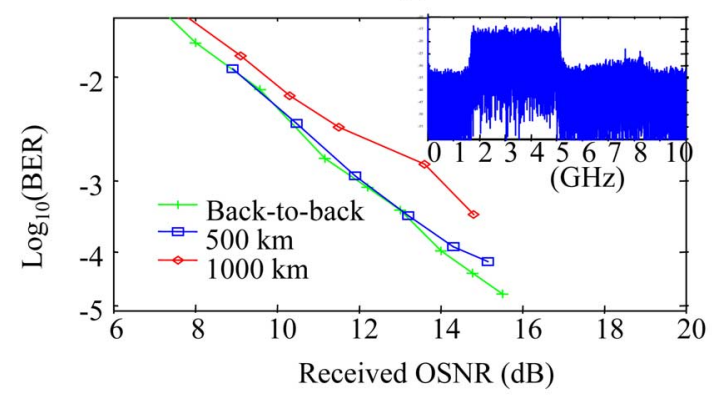

(b)

Fig. 9. (a) Q-factor and $\mathrm{Q}$ penalty versus transmission distance. The $\mathrm{Q}$ penalty curves with squares is after fiber nonlinearity mitigation. (b) System performance at 500 and $1000 \mathrm{~km}$.

timum launch power for ten loops can be roughly estimated as $-7 \mathrm{dBm}$. In the multi-span long-haul transmission, the launch power into SSMF fiber is $-6.6 \mathrm{dBm}$. Fig. 9(a) shows the Q-factor evolution with the distance up to $1000 \mathrm{~km}$. The Q-factor at the same OSNR in the back-to-back transmission is also recored as the reference to compute system penalty. The Q-factor is mainly decided by the OSNR when the transmission distance is short. However, the nonlinear phase noise occurs after $500 \mathrm{~km}$ and the Q-factor penalty at $1000 \mathrm{~km}$ becomes significant. As a result, there is an error floor at $10^{-4} \mathrm{BER}$. The improvement of the fiber nonlinearity mitigation is not apparent because the nonlinearity in the system is week due to the low launch power. Furthermore, as explained in [8], the mitigation method in this work is accurate for the single span transmission but only an approximation for the multi-span transmission. More advanced signal processing techniques are needed to mitigate the fiber nonlinearity for the multi-span transmission.

Fig. 9(b) shows the BER performance after 500- and $1000-\mathrm{km}$ transmissions. The inset is the spectrum of the OFDM signal after $1000-\mathrm{km}$ transmission. The spectrum is still tightly bounded within $3.5 \mathrm{GHz}$. The OSNR penalties at $10^{-3} \mathrm{BER}$ for $500-\mathrm{km}$ and $1000-\mathrm{km}$ transmissions are 0.1 and $2 \mathrm{~dB}$, respectively. The fiber nonlinearity mitigation results are not shown in Fig. 9(b) because the improvements are insignificant, as explained in Fig. 9(a).

\section{CONCLUSION}

Software-enhanced optical transmission (SEOT) exploits digital signal processing to enhance the performance of optical transmissions. Coherent optical OFDM (CO-OFDM) is a typical example of SEOT, showing many advantages and flexibilities due to software capabilities.

Phase noise is a main impairment for high spectral efficiency CO-OFDM systems. In this paper, a transmission model with an emphasis on digital signal processing was reviewed. It was shown that laser linewidth is an important parameter to design such systems. By using simulation, the BER floors from finite linewidth laser sources were presented. Consequently, optimum subcarrier number and M-QAM constellation point could be selected under the constraint of a specific laser linewidth. 4-QAMOFDM and 16-QAM-OFDM were shown favorable for optical fiber transmissions since they could be realized by available laser sources with lower OSNR requirements than conventional IM/DD optical transmission systems.

The phase noise effects were further investigated in two experiments, in which the effective bit rates of 16-QAM-OFDM and 64-QAM-OFDM were $10.59 \mathrm{~Gb} / \mathrm{s}(2.8 \mathrm{bit} / \mathrm{s} / \mathrm{Hz}$ spectral efficiency) and $15.89 \mathrm{~Gb} / \mathrm{s}(4.2 \mathrm{bit} / \mathrm{s} / \mathrm{Hz}$ spectral efficiency), respectively. In the back-to-back measurement, 64-QAM-OFDM was strongly limited by laser phase noise whereas 16-QAM-OFDM had little phase noise effect. In the second experiment, the nonlinear phase noise effects from optical fiber transmission were investigated for single-span and multi-span transmission. With $-6.6-\mathrm{dBm}$ launch power into the transmission fiber, the OSNR penalties at $10^{-3}$ BER for 500- and $1000-\mathrm{km}$ transmissions were 0.1 and $2 \mathrm{~dB}$, respectively. Finally, the fiber nonlinearity penalty reduction after the receiver-based signal processing was significant in the single-span transmission, but insignificant in the multi-span transmission.

\section{APPENDIX}

The SNR per symbol $R_{s}$ of coherent optical OFDM (COOFDM) can be calculated from the noise spreading of constellation points as described in [10]. The SNR per symbol and SNR per bit are related by

$$
R_{b}=\frac{R_{s}}{\log _{2} M} .
$$

In the AWGN channel, single carrier and OFDM have about the same performance in terms of SNR, and the BER of M-QAM-OFDM is given by [20], [21]

$$
\begin{aligned}
P_{e} & =\frac{1-\left[1-2\left(1-\frac{1}{\sqrt{M}}\right) Q\left(\sqrt{\frac{3 R_{s}}{M-1}}\right)\right]^{2}}{\log _{2} M} \\
Q(x) & =\frac{1}{\sqrt{2 \pi}} \int_{x}^{\infty} e^{-t^{2} / 2} d t
\end{aligned}
$$

where $M=2^{k}$ and $k$ is an even number. Note (12) is a tight upper bound of a more accurate expression in [20].

The Q-factor can be calculated from BER $P_{e}$ by

$$
\begin{aligned}
Q_{f} & =\sqrt{2} \operatorname{erf}^{-1}\left(1-2 P_{e}\right) \\
y & =\operatorname{erf}(x)=\frac{2}{\sqrt{\pi}} \int_{0}^{x} e^{-t^{2}} d t \\
x & =\operatorname{erf}^{-1}(y) .
\end{aligned}
$$




\section{REFERENCES}

[1] D. McGhan, C. Laperle, A. Savchenko, C. Li, and G. M. A. M. O'Sullivan, "5120-km RZ-DPSK transmission over G.652 fiber at $10 \mathrm{~Gb} / \mathrm{s}$ without optical dispersion compensation," IEEE Photon. Technol. Lett., vol. 18 , no. 2, pp. 400-402, Jan. 2006.

[2] A. Faerbert, "Application of digital equalization in optical transmission systems," in Conf. Optical Fiber Communication (OFC), Mar. 2006.

[3] D. S. Ly-Gagnon, S. Tsukamoto, K. Katoh, and K. Kikuchi, "Coherent detection of optical quadrature phase-shift keying signals with carrier phase estimation," J. Lightw. Technol., vol. 24, no. 1, pp. 12-21, Jan. 2006.

[4] W. Shieh, X. Yi, and Y. Tang, "Transmission experiment of multi-gigabit coherent optical OFDM systems over $1000 \mathrm{~km}$ SSMF fibre," Electron. Lett., vol. 43, no. 3, pp. 133-184, Feb. 2007.

[5] W. Shieh and C. Athaudage, "Coherent optical orthogonal frequency division multiplexing," Electron. Lett., vol. 42, no. 10, pp. 587-589, May 2006.

[6] S. L. Jansen, I. Morita, N. Takeda, and H. Tanaka, "20-Gb/s OFDM transmission over 4 160-km SSMF enabled by RF-pilot tone phase noise compensation," in Conf. Optical Fiber Communication (OFC), Mar. 2007, PDP15.

[7] X. Yi, W. Shieh, and Y. Tang, "Phase estimation for coherent optical OFDM," IEEE Photon. Technol. Lett., vol. 19, no. 12, pp. 919-921, Jun. 2007.

[8] W. Shieh, X. Yi, Y. Ma, and Y. Tang, "Theoretical and experimental study on PMD-supported transmission using polarization diversity in coherent optical OFDM systems," Opt. Expr., vol. 15, no. 16, pp. 9936-9947, Aug. 2007.

[9] Y. Tang, K. P. Ho, and W. Shieh, "Optimal design for coherent optical OFDM transmitter employing predistortion," presented at the Eur. Conf. Optical Commun. (ECOC), Sep. 2007, Paper 5.2.1.

[10] W. Shieh, R. S. Tucker, W. Chen, X. Yi, and G. Pendock, "Optical performance monitoring in coherent optical OFDM systems," Opt. Expr., vol. 15 , no. 2, pp. 350-356, Jan. 2007.

[11] S. Hara and R. Prasad, Multicarrier Techniques for 4G Mobile Communications. Boston, MA: Artech House, 2003.

[12] I. B. Djordjevic and B. Vasic, "Orthogonal frequency division multiplexing for high-speed optical transmission," Opt. Expr., vol. 14, no. 9, pp. 3767-3775, May 2006.

[13] A. J. Lowery, L. Du, and J. Armstrong, "Orthogonal frequency division multiplexing for adaptive dispersion compensation in long haul WDM! systems," in Conf. Optical Fiber Communication (OFC), Mar. 2006, PDP39.

[14] T. Okoshi and K. Kikuchi, Coherent Optical Fiber Communications. Boston, MA: Kluwer Academic, 1988.

[15] K. P. Ho and J. M. Kahn, "Electronic compensation technique to mitigate nonlinear phase noise," J. Lightw. Technol., vol. 22, no. 3, pp. 799-783, Mar. 2004.

[16] A. G. Armada, "Understanding the effects of phase noise in orthogonal frequency division multiplexing (OFDM)," IEEE Trans. Broadcasting, vol. 47, no. 2, pp. 153-159, Jun. 2001.

[17] S. Wu and Y. Bar-Ness, "OFDM systems in the presence of phase noise: Consequences and solutions," IEEE Trans. Commun., vol. 52 , no. 5, pp. 1988-1996, May 2004.

[18] T. Pollet, M. V. Bladel, and M. Moeneclaey, "BER sensitivity of OFDM: Systems to carrier frequency offset and Wiener phase noise," IEEE Trans. Commun., vol. 43, no. 2/3/4, pp. 191-193, 1995.

[19] L. Tomba, "On the effect of Wiener phase noise in OFDM systems," IEEE Trans. Commun., vol. 46, no. 5, pp. 580-583, May 1998.

[20] F. Xiong, Digital Modulation Techniques, 2nd ed. Boston, MA: Artech House, 2006.

[21] J. G. Proakis, Digital Communication, 4th ed. New York: McGrawHill, 2000.

[22] L. Hanzo and T. Keller, OFDM and MC-CDMA: A Primer. Hoboken, NJ: Wiley, 2006.

[23] E. Ip, J. M. Kahn, D. Anthon, and J. Hutchins, "Linewidth measurements of MEMS-based tunable lasers for phase-locking applications," IEEE Photon. Technol. Lett., vol. 17, no. 10, pp. 2029-2031, Oct. 2005.
[24] X. Yi, W. Shieh, and Y. Ma, "Phase noise on coherent optical OFDM systems with 16-QAM and 64-QAM beyond $10 \mathrm{~Gb} / \mathrm{s}$," presented at the Eur. Conf. Optical Commun. (ECOC), Sep. 2007, Paper 5.2.3.

[25] K. Kikuchi, "Effect of 1/f-type FM noise on semiconductor-laser linewidth residual in high-power limit," IEEE J. Quantum Electron., vol. 25, no. 4, pp. 684-688, Apr. 1989.

[26] N. S. Bergano, F. W. Kerfoot, and C. R. Davidson, "Margin measurements in optical amplifier systems," IEEE Photon. Technol. Lett., vol. 5, no. 3, pp. 304-306, Mar. 1993.

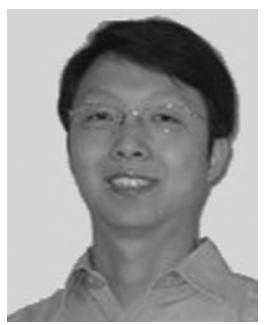

Xingwen Yi received the B.Eng. degree in electronic engineering from Southeast University, Nanjing, China, in 1999 and the Ph.D. degree in electrical and electronic engineering from the University of Melbourne, Melbourne, Australia, in 2007.

From 1999 to 2004, he was at Huawei Technologies, Co., Ltd., China. He was involved in EDFA development, system design, and key technologies investigations for DWDM long-haul transmission systems. In 2005, he was an Intern for three months at Alcatel SEL AG, Germany, where he developed algorithms for feedforward equalizers and decision feedback equalizers. He is currently a Research Scientist at the University of California, Davis. He has published more than 30 journal and conference papers. He also holds seven patents in China. His current research interests include electronic compensation of optical distortions, all-optical label swapping techniques, and optical performance monitoring.

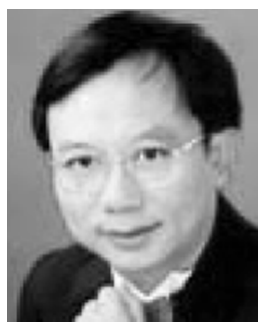

William Shieh (S'96-M'96) received the M.S degree in electrical engineering-communications and the Ph.D. degree in physics from the University of Southern California, Los Angelses, in 1994 and 1996, respectively.

From 1996 to 1998, he worked as a member of Technical Staff in the Jet Propulsion Laboratory, Pasadena, CA. From 1998 to 2000, he worked as a member of the Technical Staff in Bell Labs, Lucent Technologies, Holmdel, NJ. From 2000 to 2003, he worked as a Technical Manager in Dorsal Networks, Columbia, MD. Since 2004, he has been with the Department of Electrical and Electronic Engineering, University of Melbourne, Melbourne, Australia, as a Senior Lecturer. His current research interests include OFDM techniques in both wireless and optical communications, coherent optical communication systems, and optical packet switching. He has published more than 80 journal and conference papers, and submitted 14 U.S. patents (nine issued) covering areas of polarization controller, wavelength stabilization in WDM systems, and Raman amplifier-based systems and subsystems.

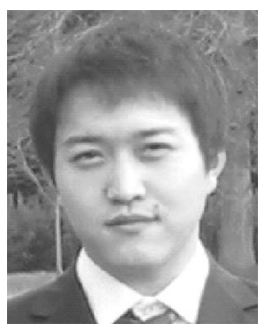

Yiran Ma received the B.Eng. degree in telecommunication engineering in 2005 from the Harbin Institute of Technology, Harbin, China, the M.Telecommun.Eng. degree from the University of Melbourne, Melbourne, Australia. He is currently working toward the Ph.D. degree at the University of Melbourne.

In 2005, he was an Intern for half a year at the China Academy of Space Technology, where he developed algorithms of real-time encryption and decryption for communication networks. His current research interests include coherent optical system design, signal processing of MIMO-OFDM, and nonlinearity in optical long-haul systems. 